(2) Open Access Full Text Article

CASE SERIES

\title{
Atypical Locations of Renal Cell Carcinoma Metastases to the Pancreas and Duodenum
}

This article was published in the following Dove Press journal:

Research and Reports in Urology

\author{
Łukasz Piskorz' \\ Kryspin Mitura $\mathbb{D}^{2,3}$ \\ Witold Olejniczak' \\ Piotr Misiak ${ }^{4}$ \\ Slawomir Jablonski ${ }^{4}$ \\ 'Department of General Surgery, \\ Brothers of St John of God Hospital, \\ Lodz, Poland; ${ }^{2}$ Department of General \\ Surgery, Siedlce Hospital, Siedlce, Poland; \\ ${ }^{3}$ Faculty of Medical and Health Sciences, \\ University of Natural Sciences and \\ Humanities in Siedlce, Siedlce, Poland; \\ ${ }^{4}$ Clinic of the Chest Surgery, General and \\ Oncological Surgery, Military Medical \\ Academy University Teaching Hospital - \\ Central Veterans' Hospital, Lodz, Poland
}

Correspondence: Kryspin Mitura Narutowicza 25, Siedlce 08-II0 Tel +48602809035

Email chirurgia.siedlce@gmail.com

\begin{abstract}
Introduction: Pancreatic tumors account for a small percentage of all malignancies. Most of them are primary and originate from cells of the exocrine pancreas. The remaining primary changes are neuroendocrine tumors. The pancreas may also be a target of metastatic lesions. The most common cancer that metastasizes to the pancreas is renal cell carcinoma.
\end{abstract}

Case Studies: The paper presents two cases of rare metastases to organs of the abdominal cavity: the first patient treated surgically due to two metastatic lesions of renal cell carcinoma in the pancreas, diagnosed many years after radical nephrectomy. The second case of high gastrointestinal obstruction in the course of metastasis of renal cell carcinoma to the duodenum. The first patient underwent distal laparotomic resection of the pancreas. The second patient underwent resection of the duodenum and the first jejunal loop with sideto-side duodeno-jejunal anastomosis. Both patients remain under oncological supervision.

Conclusion: Patients after radical nephrectomy due to renal cell carcinoma require longterm systematic monitoring. Due to the anatomical position of the pancreas and duodenum as well as the number, location and size of metastatic lesions, the course of the disease may be initially asymptomatic or oligosymptomatic. Aggressive surgical treatment of pancreatic metastases creates opportunities for long-term survival.

Keywords: renal cell carcinoma, metastases, pancreas, duodenum, surgery

\section{Introduction}

Primary pancreatic tumors account for a small proportion of all malignancies (approximately 2\%). Most of them, as many as 95\%, originate from cells of the exocrine part - lining the pancreatic ducts. The remaining changes are tumors originating in the endocrine pancreas -neuroendocrine tumors (NETs). NETs can be active, producing and secreting hormones that cause certain symptoms, or hormonally inactive, which applies to most lesions. The pancreas is an organ in which metastatic lesions can also be located, but this phenomenon is rare. The most common cancer that metastasizes to the pancreas is renal cell carcinoma (RCC). ${ }^{1,2} \mathrm{RCC}$ accounts for $2-3 \%$ of adult malignancies. The course of the disease is unpredictable, which may be the result of various pathways and mechanisms of cancer dissemination. ${ }^{3}$ At diagnosis, metastatic changes are found in $20-30 \%$ of patients, and in another $40-50 \%$ dissemination occurs later. ${ }^{4}$ Most often they are multiple. Single metastasis occurs only in $1-3 \%$ of patients. ${ }^{5,6}$ RCC metastases are most often located in the lungs, bones, liver, brain, and adrenal glands, while localization in other organs such as the pancreas, thyroid, or large intestine is rare. ${ }^{7-11}$ On the other hand, a review of the literature indicates that RCC is the first among those that metastasize 
to the pancreas, which was emphasized earlier. ${ }^{1,2}$ In contrast, duodenal metastases are very rare. The method of treating RCC depends on the clinical stage. The existence of multiple synchronous metastases in other organs may limit the benefit of surgical resection of the pancreatic metastases; therefore, radical surgical treatment may be considered primarily in solitary resectable lesions. Molecular methods and immunotherapy find their place in more complex cases. The recent introduction into the clinical practice of novel therapies (ie, tyrosine kinase inhibitors and rapamycin inhibitors) may significantly improve outcomes. $^{3}$ In the case of local recurrence of the disease or limited single-organ metastases, surgical treatment is applicable, provided that the general condition of the patient and coexisting disease allow it. This therapeutic option is rather recommended for patients who had recurrences many years after the primary treatment, as early recurrences often present as multiple lesions. ${ }^{4,5}$ The role of radiotherapy and chemotherapy is limited. ${ }^{3}$
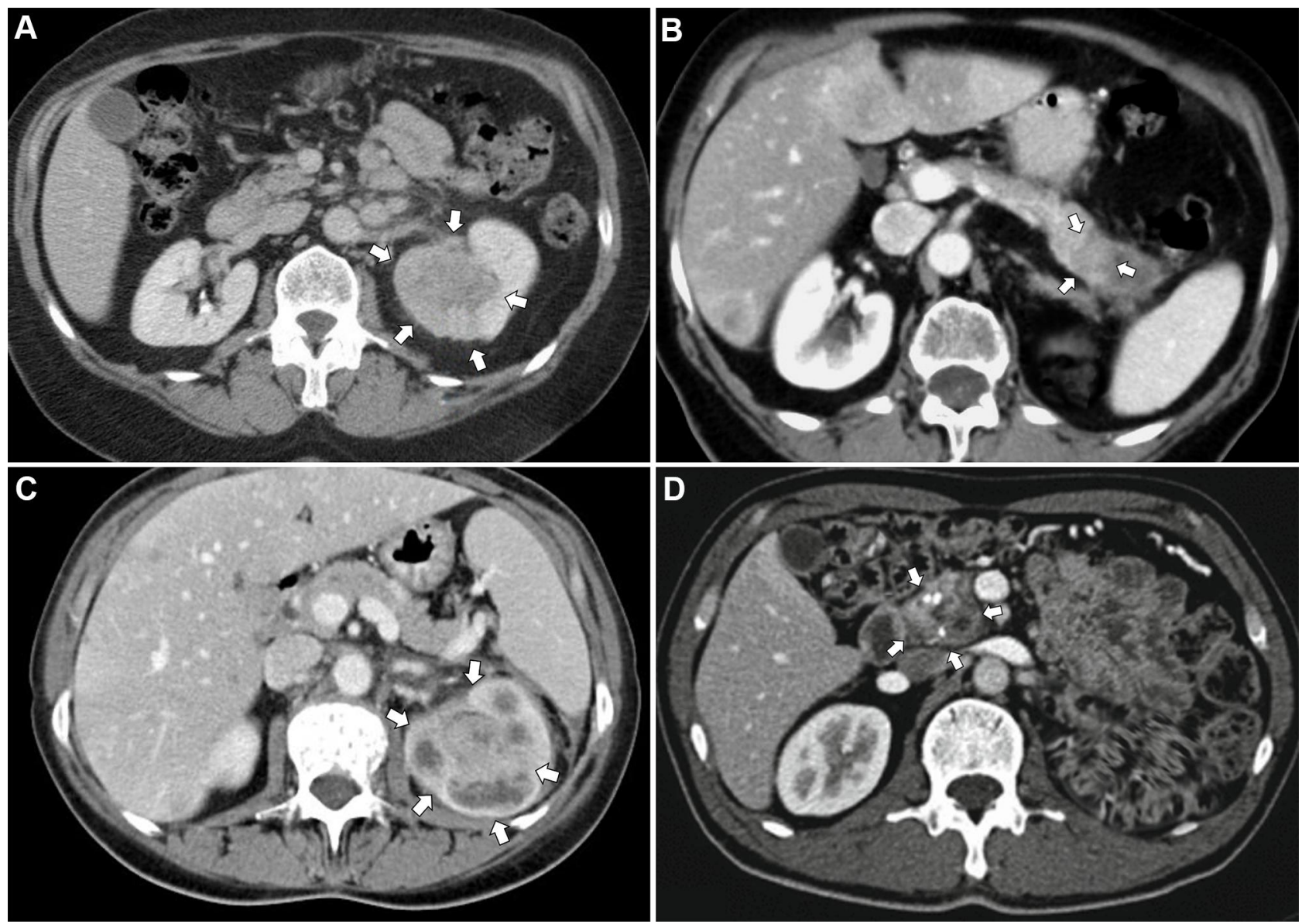

Figure I Computed tomography image of a primary RCC tumor in the left kidney (A) and metastatic lesion in a tail of the pancreas (B) in the first presented case. A primary tumor of the left kidney $(\mathbf{C})$ and metastatic lesion of the duodenum (D) in the second case. Pathologic lesions have been marked with arrows. 
abdominal pain and weight loss due to vomiting $-11 \%$ within 4 months. The examination revealed two focal lesions in the pancreas, intensely enhanced in the arterial phase. The first lesion, 20x15 mm in size, was located on the border of the body and the tail of the pancreas, the second was $10 \mathrm{~mm}$ in diameter, located in the tail. There was no evidence of vascular infiltration or enlargement of regional lymph nodes. X-ray of the chest showed no metastatic changes. The patient was qualified for surgery due to the possibility of total resection of small lesions with no concomitant metastases. The abdomen was opened by a horizontal laparotomy in the left upper abdomen. Review of the abdominal cavity showed no signs of cancer dissemination. Numerous adhesions of the greater omentum and loops of the small intestine with the parietal peritoneum were found in the abdominal cavity. In the distal part of the body and in the tail of the pancreas, there were two tumor-like lesions. The pancreas was in adhesions with surrounding tissues. During the surgery, a distal pancreatic resection was performed in the area of the body and tail. The patient was discharged home in good condition on the tenth day after the surgery. Currently, two years later, the patient remains under oncological supervision. No signs of recurrence have been observed.

\section{Histopathological Examination}

The pancreas measured $16.5 \mathrm{~cm} \times 3.5 \times 2 \mathrm{~cm}$. A solid circular tumor, $15 \mathrm{~mm}$ in diameter on the cross-section within the body. Within the tail, a beige tumor, $8 \mathrm{~mm}$ in diameter. The tumor did not reach the surgical incision line, and five removed lymph nodes were free from cancer invasion. Microscopic image and immunohistochemical profile of tumors (IHC: CD10 +, vimentin focally + ) corresponded to metastases of clear cell RCC (Figure 2). In all pictures, the hematoxylin + eosin stained tumor showed a structure composed of clear cells with a centrally located nucleus - typical of clear cell RCC. The tumor formed solid metastatic lesions that were quite well isolated from the pancreatic parenchyma. Figure 3 shows the active infiltration of the surroundings.

\section{Case Study 2}

A 76-year-old female patient was admitted to the hospital due to confirmed and histopathologically verified metastasis of clear cell RCC to the duodenum, causing nearly complete gastrointestinal obstruction (Figure 1D). Additionally, the patient reported weight loss by $12 \mathrm{~kg}$ in the previous three months, which was $14 \%$ of the initial body weight (current BMI 31.6). In medical history: a radical nephrectomy due to clear cell RCC of the left kidney (Fuhrman malignancy stage 2) eight years earlier (Figure 1C). After the surgery, the patient was not qualified for any additional therapy. The patient was reoperated twice due to local recurrence of the disease (one year and then four years after the primary resection). Four years later (eight years after the first intervention), the patient developed abdominal pain in the epigastrium and midabdomen, nausea, and periodic vomiting with hyme. Control imaging revealed a tumor-like lesion in the duodenum. The PET-CT examination with intravenous administration of the radiopharmaceutical 18F-choline with (activity of $192 \mathrm{Mbq}$ ) demonstrated a status after left nephrectomy - without a presence of metabolically active foci in the surgical bed. In the distal part of the duodenum - regularly distended - there was a mass of $5.4 \times 6.9$ x $6.8 \mathrm{~cm}$, showing increased metabolism: SUVmax Fch = 8.1. No other metabolically active foci were found. In CT, there was no evidence of recurrence within the surgical bed. Compared to the examination performed 6 months earlier, segmental dilatation of the duodenum was found in front of the Treitz ligament with a polypoid, heterogeneous central mass, strongly enhanced, dimensions of 64 x $47 \times 41-$ a recurrence of the neoplastic process in the form of metastases to the duodenum. Endoscopic examination showed a lumpy, polycyclic, fragile lesion located in the duodenum behind the bulb and filling its lumen. On the basis of the histopathological examination of the collected specimens, recurrence of RCC was confirmed: focally present clear cells with $\mathrm{CD} 10+$ immunophenotype present in the granulation tissue, RCC unreliable - no material from the lesion. $\mathrm{CKAE}+, \mathrm{CD} 68$ focally+, CD31 + in vessels, S100-, synaptophysin-. The presentation most closely corresponded to the metastasis of clear cell RCC (Figure 2). The patient was qualified for surgical treatment due to increasing mechanical obstruction of the duodenum. Laparotomy was performed by an upper median incision. Review of the abdominal cavity showed no signs of cancer dissemination. In the retrobulbar part of the duodenum, the tumor was filling the lumen of the organ over a distance of about $6-8 \mathrm{~cm}$ and ended in the area of the duodenum suspensory ligament. The described lesion did not extend beyond the organ wall. The duodenum and the first jejunum loop were resected. Continuity of the gastrointestinal tract was restored by side-to-side duodeno-jejunal anastomosis. The postoperative course 


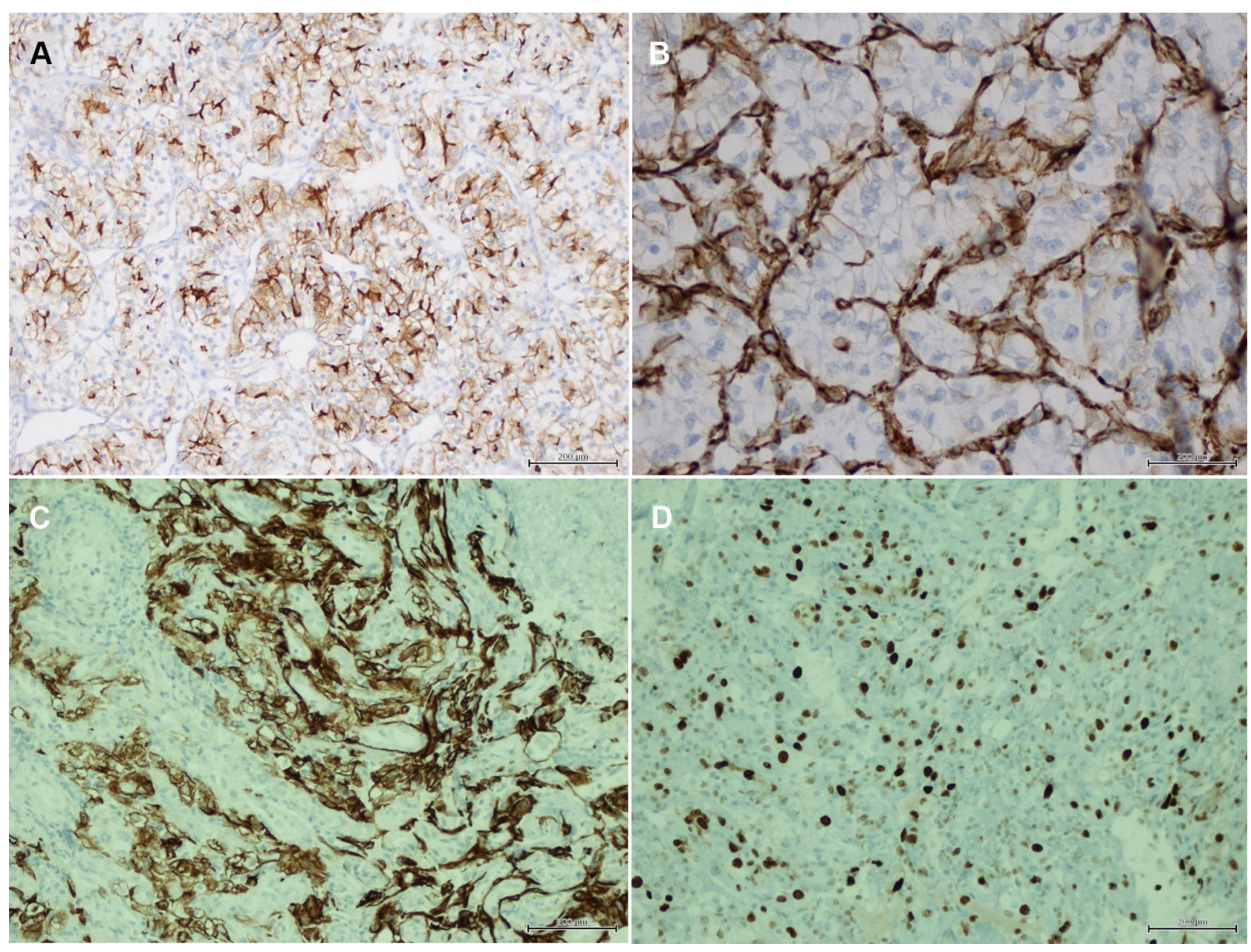

Figure 2 CDI0 immunohistochemistry confirms clear cell RCC histogenesis as a metastasis of clear cell carcinoma from the primary kidney tumor (A). Vimentin immunohistochemistry in the form of colored reactions confirms the cancer histogenesis (B). Immunohistochemical reaction to the presence of cytokeratins in cancer cells (C). The immunohistochemical reaction with the Ki67 antibody shows high mitotic activity of cancer cells (D).

was uneventful. The patient was discharged home in good condition on the ninth postoperative day. Two years passed since that treatment and the patient remains under constant oncological supervision with no signs of recurrence.

\section{Histopathological Examination}

Clear cell RCC of the second stage of malignancy according to Fuhrman - a metastasis of RCC.

A fragment of duodenum and jejunum $14 \mathrm{~cm}$ long with an exophytic tumor measuring $7 \times 5.5 \times 2.5 \mathrm{~cm}$ (proximal margin $-3.5 \mathrm{~cm}$, distal $-3 \mathrm{~cm}$, radial margin macroscopically $0.1 \mathrm{~cm})$. Tumor infiltration included the mucosa, submucosa, muscularis, and subserous adipose tissue of the small intestine. The tumor base vessels did not contain emboli of tumor cells. Surgical incision lines and intestinal wall outside the tumor were free of cancer infiltration (Figure 3).

\section{Discussion}

Metastatic changes in the pancreas are rare, accounting for $2 \%$ to $5 \%$ of all malignancies of this organ. ${ }^{1,2}$ Cancers most often mentioned as metastasizing to the pancreas include RCC, melanoma, colorectal cancer, breast cancer, and sarcomas. The duodenum is an even rarer localization of metastatic lesions.

RCC is an unpredictable oncological disease, often recurring in the form of single or multiple metastatic focal lesions, even many years after a radical surgical treatment. This proves the individual variability of the disease. The most common targets of metastases are lungs, bones, liver, nephrectomy bed, and brain. Pancreas, duodenum, ovary, tonsils, thyroid gland, esophagus, diaphragm, testicle, adrenal glands, and soft tissue of the knee are rare sites of RCC metastases. ${ }^{12}$ Metastases are also located in the adrenal glands and may be of synchronic or metachronic character. ${ }^{13}$ 


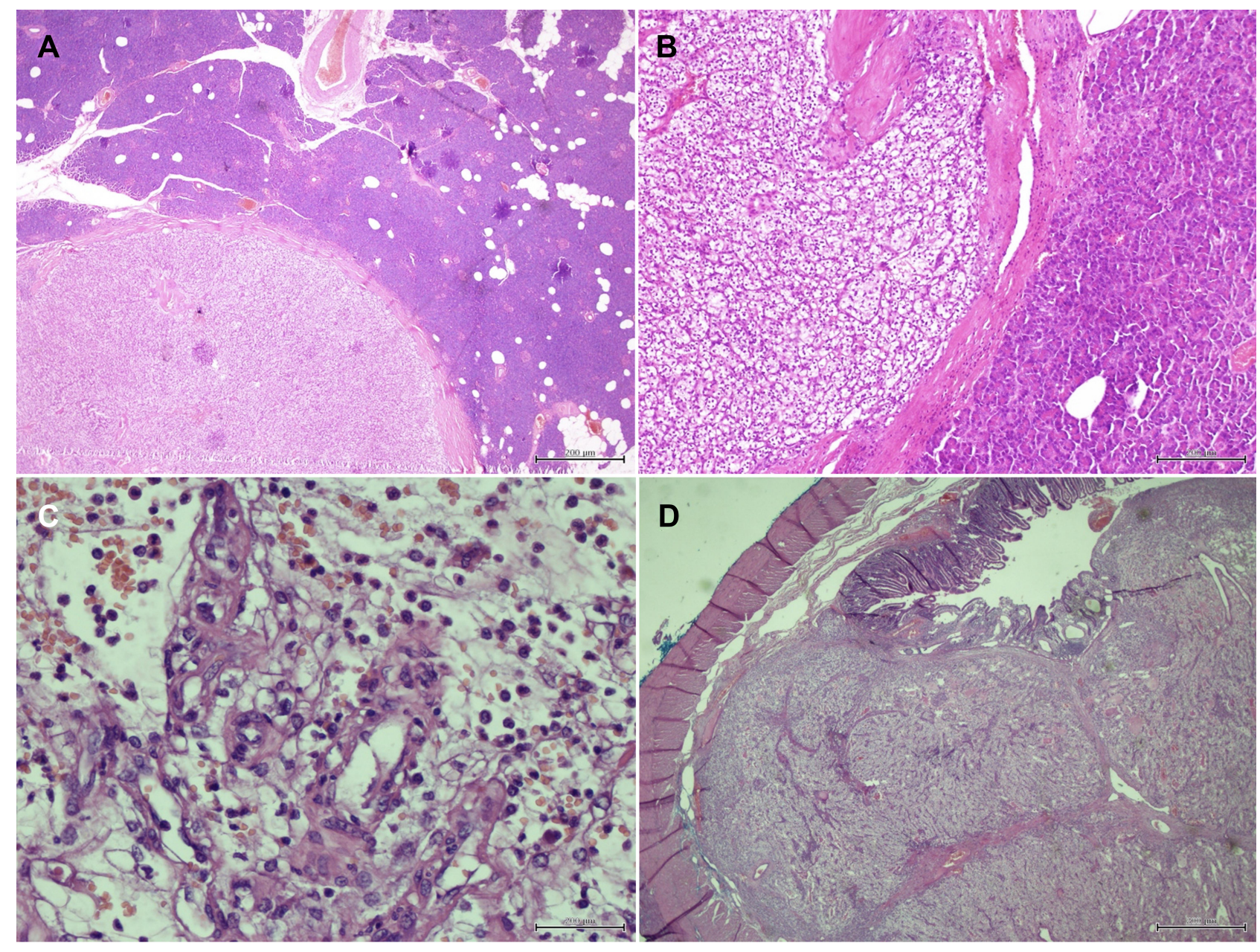

Figure 3 The pancreas with hematoxylin and eosin $(\mathrm{H}$ and $\mathrm{E})$ stained tumor $(\mathbf{A})$. The tumor in the hematoxylin and eosin staining shows a structure composed of clear cells with centrally located nuclei (B). Typical microscopic picture of clear cell carcinoma; H + E staining (hematoxylin, eosin) magnification 400 (C). HE staining, the ratio of tumor to the duodenal wall and mucosa (D).

It is worth emphasizing that recurrences of the neoplastic process in the form of metastases are found at different stages of advancement of the primary lesion. ${ }^{1,14-17}$ In the literature, on the subject there are case reports, the authors of which prove that they recorded recurrences and metastases both in patients with the first and third stage of the disease according to Robson. ${ }^{1}$ The time that elapses from the resection of the primary tumor to the diagnosis of recurrence is highly variable, often very long. ${ }^{7,13}$ The onset of pancreatic metastases in RCC usually occurs quite late, with a median of about eight years after nephrectomy. ${ }^{4}$ Antonelli et al reported the metachronous metastases diagnosed at a range of 8-73 months after renal surgery. ${ }^{13}$ In the literature, however, both descriptions of early recurrences up to a year after radical nephrectomy, and extremely late recurrences occurring as late as 27 years after the primary surgery can be found. ${ }^{15}$ A relationship between the number of pancreatic metastases and treatment outcomes has not been confirmed, and the number of lesions has no significant prognostic significance. ${ }^{4,17,18}$ In the presented case, recurrence of the disease in the pancreas was found seven years after nephrectomy, and the primary disease was stage 2 . The late appearance of pancreatic lesions fits within the time ranges presented in the literature, and the low advancement of the primary tumor confirms the unpredictable course of the cancer reported in the literature. Despite the fact that the diagnosis of metastatic RCC indicates a generalized nature of the disease, metastatic RCC may be an isolated recurrence, which is often associated with longer survival after aggressive surgical treatment. The literature describes cases of metachronic recurrence after previous resection of liver metastases and cases of simultaneous synchronous metastases of RCC to the pancreas 
and thyroid gland, adrenal gland or pituitary gland. ${ }^{19-21}$ The second presented case confirms the observations made by other authors. The patient we operated on had previously undergone two surgical treatments due to recurrence in the surgical bed of the removed kidney. The survival time of patients after metastasectomy due to metachronic and synchronic pancreatic metastases is similar. Metastases to regional lymph nodes and infiltration of vessels and adjacent organs are associated with a worse prognosis. ${ }^{17}$

The presence of RCC metastases in both typical and rare locations: pancreas, duodenum, or early stage ovaries, depending on the size, location, and number, is initially asymptomatic. ${ }^{22}$ Disease recurrence is most often found during routine checkups. In other cases, the course is mildly symptomatic or noncharacteristic, with nonspecific abdominal pain, symptoms of impaired gastrointestinal patency, jaundice, and other symptoms associated with gastrointestinal pathology or ovarian tumor. ${ }^{16,23,24}$ Weight loss is usually a common symptom of concern. In both presented cases, weight loss in the 3 months prior to admission to hospital exceeded $10 \%$ of the baseline body weight. The clinical presentation is often a consequence of the location of the tumor and its size. Involvement of the head of the pancreas may result in mechanical jaundice or gastrointestinal obstruction due to duodenal compression, and may also be the result of a local process in the duodenum. ${ }^{14,24}$ In the presented case, the patient was hospitalized in the gastroenterology department in the period preceding the diagnosis due to an episode of acute pancreatitis. After the patient was discharged home, complaints appeared in the form of epigastric pain. Initially, the symptoms did not bother the patient despite the episode of pancreatitis. Only when she noticed loss of body weight she did report to the doctor, which confirms the fact that weight loss is usually not underestimated by patients and perceived as a negative factor. In the second case, the disease was confirmed in control tests, but it coincided with the appearance of upper gastrointestinal symptoms.

Among the diagnostic methods used for the detection of pathological changes in the pancreas, abdominal sonography as a screening method, computed tomography, magnetic resonance imaging, and in doubtful cases, endoscopic ultrasound (EUS) for changes located in the head of the pancreas, should be mentioned. The latter method, in addition to visualizing the lesion, also enables histopathological verification by targeted biopsy. ${ }^{25,26}$ In addition, in case of abdominal pain, nonspecific symptoms from the upper gastrointestinal tract, or, for example, an ovarian tumor in patients who have undergone treatment for $\mathrm{RCC}$, a thorough diagnostic is necessary, especially when the patient reports weight loss.

The scope and nature of the surgical treatment depend on the location of the lesion, local advancement, infiltration of adjacent organs, and the patient's general condition because in some cases resection of metastases is associated with a very extensive surgical procedure. However, in presented cases, the decision of surgical intervention has been made based upon two different indications for both patients. The first patient was qualified for surgery after an oncological advisory board meeting, due to a high possibility of a total resection of two small peripheral pancreatic lesions with no concomitant metastases. A second patient was qualified for surgical treatment due to an increasing risk of the complete mechanical obstruction of the duodenum with no additional lesions found in PET imaging. Additionally, we were concerned about an early jaundice occurrence (due to tumor location) that might restrain the use of additional therapeutic means. The lack of synchronous metastases in other organs allowed us to expect the benefit of surgical resection of the pancreatic metastases. According to Moletta ${ }^{22}$ surgery with radical intent is often the only chance of cure. Masetti et $\mathrm{al}^{27}$ presented a survival of seven years after pancreatic resection, while patients not qualified for surgical treatment had a median survival of less than a year. Recent therapies show significant outcomes; however, most of them are targeted towards multiple lesions. Therefore, solitary metastatic lesions are still considered as an indication for a surgical resection as these new therapies are not always available or affordable in some countries. Recurrence of the disease in the head of the pancreas, hepatoduodenal papilla, or the proximal section of the duodenum requires pancreatoduodenectomy. ${ }^{23,28}$ However, this extensive surgical intervention is correlated with longer survival, as emphasized by some authors. ${ }^{22}$ When metastases are located in the body or tail, the applied procedure is usually a distal pancreatic resection. Multiple locations of malignancy may require resection of the entire pancreas. In the presented case, due to the location of the lesions, a distal pancreatic resection was performed. In the postoperative course, we noted a septic condition resulting from inflammatory and atelectatic changes in the lungs and a small cistern in the operated area. The use of antibiotic therapy made it possible to control the infection. In the second 
case, localization of the lesion in the extracapsular part of the duodenum called for its resection with side-to-side anastomosis. In our opinion, it was a good solution, saving the patient from an extensive pancreatoduodenectomy.

\section{Conclusions}

Patients after radical nephrectomy due to renal cell carcinoma require long-term, regular monitoring. Due to the anatomical position of the pancreas and duodenum as well as the number, location, and size of metastatic lesions, the course of the disease may be initially asymptomatic or oligosymptomatic. Aggressive surgical treatment of pancreatic metastases gives a chance for long-term survival.

\section{Acknowledgment}

Written informed consents for publication of the clinical details and accompanying images were obtained from the patients. Institutional approval was required to publish the case details. The Ethics Committee of the Military Medical Academy University had approved the study protocol.

\section{Funding}

We are reporting this case for academic purposes and have received no external funding in support of our work.

\section{Disclosure}

The authors report no conflicts of interest for this work.

\section{References}

1. Eidt S, Jergas M, Schmidt R, et al. Metastasis to the pancreas - an indication for pancreatic resection? Langenbecks Arch Surg 2007;392:539-542. doi:10.1007/s00423-007-0148-7

2. Hegerova L, Griebeler ML, Reynolds JP, et al. Metastasis to the thyroid gland. Report of a large series from the Mayo Clinic. Am $J$ Clin Oncol. 2015;38(4):338-342. doi:10.1097/COC.0b013e318 29d1d09

3. Escudier B, Porta C, Schmidinger M, et al. Renal cell carcinoma: ESMO clinical practice guidelines for diagnosis, treatment and follow-up. Ann Oncol. 2014;25(3):11149-11156. doi:10.1093/annonc/ mdu259

4. Zerbi A, Ortolano E, Balzano G, et al. Pancreatic metastasis from renal cell carcinoma: which patients benefit from surgical resection? Ann Surg Oncol. 2008;15:1161-1168. doi:10.1245/s10434-007-9782-0

5. Haertig A, Kuss R Clinical signs in renal neoplasia. A comparation of two series of three hundred cases. Renal Tumors - Proceedings of the First International Symposium on Kindey Tumors; New York, Liss; 1982:337.

6. Tolia BM, Whitmore WF Jr. Solitary metastasis from renal cell carcinoma. J Urol. 1975;114:836. doi:10.1016/s0022-5347(17)671 $55-6$

7. Antonelli A, Arrighi N, Corti S, et al. Surgical treatment of atypical metastasis from renal cell carcinoma. BJU Int. 2012;110:E559-E563. doi:10.1111/j.1464-410X.2012.11271.x

8. Koszarowski T. Onkologia Kliniczna. Warszawa: PZWL; 1985.
9. Ritchie AWS, Chisholm GD. The natural history of renal carcinoma. Semin Oncol. 1983;10(4):390.

10. Zieliński J. Onkologia Urologiczna. Warszawa: PZWL; 1986.

11. Vo E, Palacio CH, Omino R, et al. Solitary colon metastasis from renal cell carcinoma nine years after nephrectomy: a case report. Int J Surg Case Rep. 2016;27:55-58. doi:10.1016/j.jjscr.2016. 07.053

12. Arroyo C, Palacios P, Uribe N, et al. Uncommon metastases in renal carcinoma. Gac Med Mex. 2005;141:543-546.

13. Antonelli A, Cozzoli A, Simeone A, et al. Surgical treatment of adrenal metastasis from renal cell carcinoma: a single-centre experience of 45 patients. bju int. 2006;97:505-508. doi:10.1111/j.1464410X.2006.05934.X

14. Kassabian A, Stein J, Jabbour N, et al. Renal cell carcinoma metastatic to the pancreas: a single-institution series and review of the literature. Urology. 2000;56:211-215. doi:10.1016/s0090-4295(00) 00639-7

15. Chi TY, Sang XT, Mao YL, et al. [Diagnosis and treatment of pancreatic metastasis from renal cell carcinoma]. Zhonghua Zhong Liu Za Zhi. 2008;30(10):793-796 [Article in Chinese]

16. Valappil SV, Toon PG, Anandaram PS. Ovarian metastasis from primary renal cell carcinoma: report of a case and review of literature. Gynecol Oncol. 2004;94:846-849. doi:10.1016/j.ygyno. 2004.06.035

17. Fikatas P, Klein F, Andreou A, et al. Long-term survival after surgical treatment of renal cell carcinoma metastasis within the pancreas. Anticancer Res. 2016;36:4273-4278.

18. Reddy S, Edil BH, Cameron JL, et al. Pancreatic resection of isolated metastases from nonpancreatic primary cancers. Ann Surg Oncol. 2008;15:3199-3206. doi:10.1245/s10434-008-0140-7

19. Sellner F, Tykalsky N, De Santis M, et al. Solitary and multiple isolated metastases of clear cell renal carcinoma to the pancreas: an indication for pancreatic surgery. Ann Surg Oncol. 2006;13:75-85. doi:10.1245/ASO.2006.03.064

20. Wu C, Zhou Z, Ye X, et al. Synchronous renal cell carcinoma metastasis to the contralateral adrenal gland and pancreas: a case report with 7-year follow-up subsequent to surgical therapy. Oncol Lett. 2016;11:4144-4146. doi:10.3892/ol.2016.4510

21. Kanthan GL, Schembri GP, Samra J, et al. Metastatic renal cell carcinoma in the thyroid gland and pancreas showing uptake on 68Ga DOTATATE PET/CT scan. Clin Nucl Med. 2016;41:583-584. doi:10.3892/ol.2016.4510

22. Moletta L, Milanetto AC, Vincenzi V, et al. Pancreatic secondary lesions from renal cell carcinoma. World J Surg. 2014;38:3002-3006. doi:10.1007/s00268-014-2672-2

23. Adamo R, Graeney PJ Jr, Witkiewicz A, et al. Renal cell carcinoma metastatic to the duodenum: treatment by classic pancreaticoduodenectomy and review of the literature. $J$ Gastrointest Surg. 2008;12:1465-1468. doi:10.1007/s11605-007-0426-2

24. Bhatia A, Das A, Kumar Y, et al. Renal cell carcinoma metastasizing to duodenum: a rare occurrence. Diagn Pathol. 2006;1:29. doi:10.1186/1746-1596-1-29

25. Pannala R, Hallberg-Wallace KM, Smith AL, et al. Endoscopic ultrasound-guided fine needle aspiration cytology of metastatic renal cell carcinoma to the pancreas: A multi-center experience. Cytojournal. 2016;13:24. doi:10.4103/1742-6413.192191

26. Boussios S, Zerdes I, Batsi O, et al. Pancreatic resection for renal cell carcinoma metastasis: an exceptionally rare coexistence. J Surg Case Rep. 2016;27:198-201. doi:10.1016/j.ijscr.2016.08.039

27. Masetti M, Zanini N, Martuzzi F, et al. Analysis of prognostic factors in metastatic tumors of the pancreas: a single-center experience and review of the literature. Pancreas. 2010;39(2):135-143. doi:10.1097/ MPA.0b013e3181bae9b3

28. Omranipour R, Zadeh HM, Ensari F, et al. Duodenal metastases from renal cell carcinoma presented with melena: review and case report. Iran J Pathol. 2017;12:272-276. doi:10.30699/ijp.2017.25687 


\section{Publish your work in this journal}

Research and Reports in Urology is an international, peer-reviewed, open access journal publishing original research, reports, editorials, reviews and commentaries on all aspects of adult and pediatric urology in the clinic and laboratory including the following topics: Pathology, pathophysiology of urological disease; Investigation and

Submit your manuscript here: https://www.dovepress.com/research-and-reports-in-urology-journal treatment of urological disease; Pharmacology of drugs used for the treatment of urological disease. The manuscript management system is completely online and includes a very quick and fair peer-review system, which is all easy to use. Visit http://www.dovepress.com/ testimonials.php to read real quotes from published authors. 